\title{
Mean Temporal Spatial Isochrones Direction as Marker for Activation Delay in Patients with Arrhythmogenic Cardiomyopathy
}

\author{
Rob Roudijk ${ }^{1,2}$, Peter Loh $^{2}$, Peter van Dam ${ }^{3,4}$ \\ ${ }^{1}$ Netherlands Heart Institute, Utrecht, the Netherlands, ${ }^{2}$ UMC Utrecht, Utrecht, the Netherlands, \\ ${ }^{3}$ PEACS, Nieuwerbrug, the Netherlands, ${ }^{4}$ Luzern University, Luzern, Switzerland
}

\begin{abstract}
Patients with arrhythmogenic cardiomyopathy (ACM) often present with ventricular arrhythmias due to local activation delay. Early detection of activation delay could aid in preventing sudden cardiac death. The aim of this feasibility study is to use a novel ECG analysis method, the mean temporal spatial isochrones (meanTSI), as marker of activation delay.

We included 16 patients with symptomatic ACM, 6 asymptomatic ACM and 6 controls. The standard 12 lead ECG and a standard heart model were used to construct the meanTSI $25 \mathrm{~ms}$ after the end of the QRS complex. Asymptomatic mutation carriers had significantly more meanTSI movement in the basal plane compared to Controls and ACM patients with prolonged QRS duration.

This meanTSI algorithm might become a novel screening method to quantify the activation delay in patients with ACM and asymptomatic mutation carriers in a non-invasive manner.
\end{abstract}

\section{Introduction}

Arrhythmogenic cardiomyopathy (ACM) is an inherited disease which is characterised by local activation delay due to myocardial fibrosis and fibrofatty replacement in the right free wall. [1] Activation delay can result in re-entry induced ventricular arrhythmias (VA). Often, patients with ACM have VA or sudden cardiac death as first presentation of the disease. Asymptomatic mutation carriers of a pathogenic mutation for ACM and patients with ACM are frequently followed at dedicated cardio-genetic outpatient clinics. Serial diagnostic tests are performed to detect disease progression. Early recognition of ACM and activation delay is essential to prevent sudden cardiac death. Currently, only the ECG or an invasive electrophysiological study (EPS) can be used to asses local activation delay. [2] The ECG can be used as screening tool but lacks the ability to relate the electrical signals to the cardiac anatomy.
Novel ECG techniques can combine ECG data with cardiac anatomy. For example, the mean temporal spatial isochrones (meanTSI) are derived from the vector cardiogram (VCG). The computation of the VCG from the recorded 12 lead ECG takes into account the electrode positions on the thorax. The VCG represents the mean direction of cardiac activation over time. Assuming an His-Purkinje activation, the activation starts on the left side of the septum. The meanTSI is a computation of the mean position of the cardiac activation, with the left septum as starting point. [3] Under normal circumstances the myocardial activation ends at the end of the QRS, but in patients with delayed activation in the right ventricle this might be different.

\subsection{Aim of the study}

The aim of the study is to use the ECG derived meanTSI as a marker for local activation delay. Early detection of local activation delay could possibly differentiate patients with early stages of ACM from asymptomatic mutation carriers. We hypothesized that in patients with ACM the meanTSI is directed towards the right ventricle in the $25 \mathrm{~ms}$ after the end of the QRS.

\section{Methods}

\subsection{Patient Selection}

We selected 16 consecutive patients who fulfilled the 2010 Task Force Criteria for ACM [4] and 6 asymptomatic pathogenic PKP2 mutation carriers from a dedicated cardio-genetic outpatient clinic. As a control group we used 6 patients that visited the outpatient clinic for screening of heart disease. Patients and controls underwent an ECG, echocardiogram, 24 hour holter and exercise testing. In all controls heart disease was excluded. Standard 12-lead ECG was available for all patients and controls (GE Healthcare, Milwaukee, WI, USA, MAC® $5500 \mathrm{HD}$, filter setting at $150 \mathrm{~Hz}$ ). All baseline characteristics are presented in table 1 . 


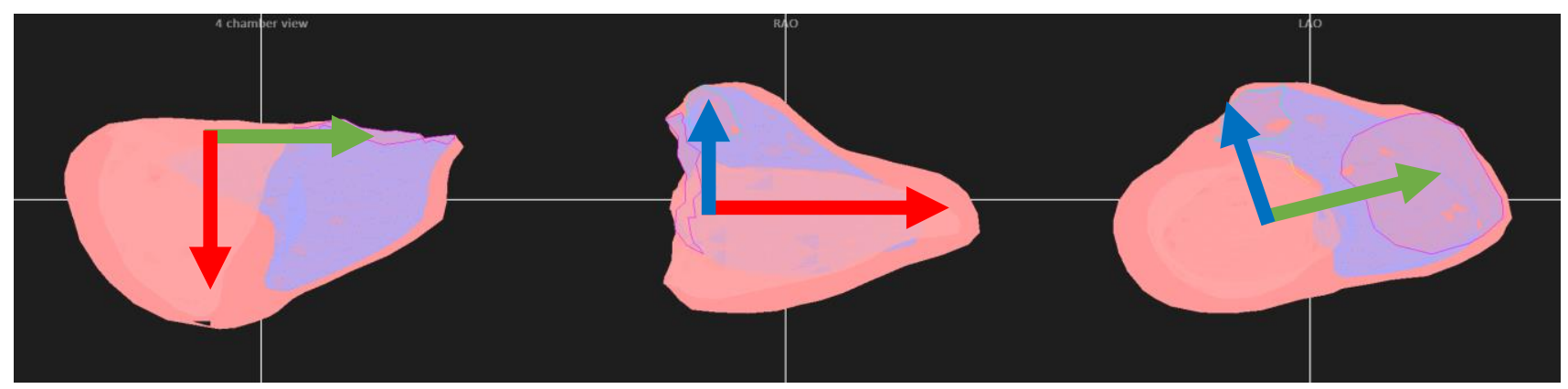

Figure 1: the left ventricle is projected in red and the right ventricle in blue. The 4 chamber view (left panel), Right Anterior Oblique (RAO) view (middle panel) and the Left Anterior Oblique view (LAO) also referred to as the basal plane of the heart (right panel). The green arrow shows the Left-Right axis (LR-axis), the blue arrow the posterioranterior axis (PA-axis), and the red arrow the base-apex axis (BA-axis).

Table 1. Baseline characteristics of the 4 patient groups

\begin{tabular}{|c|c|c|c|c|}
\hline $\begin{array}{l}\text { Paramet } \\
\text { er }\end{array}$ & $\begin{array}{l}\text { Carrier } \\
(n=6)\end{array}$ & $\begin{array}{c}\text { ACM } \\
\text { QRS < 120 } \\
(\mathrm{n}=10)\end{array}$ & $\begin{array}{c}\text { ACM } \\
\text { QRS > 120 } \\
(\mathrm{n}=6)\end{array}$ & $\begin{array}{c}\text { Control } \\
(\mathrm{n}=6)\end{array}$ \\
\hline Age & $32 \pm 17$ & $42 \pm 16$ & $59 \pm 13$ & $38 \pm 15$ \\
\hline Female & $3(50)$ & $6(60)$ & 1(17) & $2(33)$ \\
\hline $\begin{array}{l}\text { VT/ } \\
\text { SCD }\end{array}$ & 0 & $5(50)$ & $4(67)$ & 0 \\
\hline ICD & 0 & $6(60)$ & $5(83)$ & 0 \\
\hline $\begin{array}{l}\text { PR int } \\
(\mathrm{ms})\end{array}$ & $146 \pm 11$ & $147 \pm 15$ & $193 \pm 17$ & $\begin{array}{c}159 \pm \\
17\end{array}$ \\
\hline $\begin{array}{l}\text { QRS dur } \\
\text { (ms) }\end{array}$ & $94 \pm 18$ & $96 \pm 7$ & $151 \pm 14$ & $91 \pm 5$ \\
\hline $\begin{array}{l}\mathrm{PVC} \\
/ 24 \mathrm{~h}\end{array}$ & 33 & 3692 & 3397 & 109 \\
\hline \multicolumn{5}{|c|}{$\begin{array}{l}\text { Asymptomatic mutation carrier } \\
\text { arrhythmogenic cardiomyopathy (ACM), and Control } \\
\text { group. VT: Ventricular Tachycardia, SCD: Sudden } \\
\text { Cardiac Death, ICD: Implantable Cardioverter } \\
\text { Defibrillator, PVC: Premature Ventricular Complex. } \\
\text { Numbers in brackets are } \% \text {. }\end{array}$} \\
\hline
\end{tabular}

\subsection{Mean TSI algorithm}

The vector cardiogram (VCG) is a standard clinical tool to visualize the cardiac activation in 3D space. Several problems arise from this $3 \mathrm{D}$ representation, first the trajectory is relative to an arbitrary origin, second the $\mathrm{x}, \mathrm{y}, \mathrm{z}$ directions are in torso orientation and therefore make the interpreter relate these $\mathrm{x}, \mathrm{y}$, and $\mathrm{z}$ signals to the cardiac anatomy. In the proposed method a standard model of the heart and torso is used to compute and visualize this relation.

The first step is to compute the VCG from the ECG signals and the electrode positions on the thorax. In this study the VCG was derived from the ECG while taking the electrode positions into account. The $\overrightarrow{V C G}$ is computed from the 9 electrodes, building the 12 lead
ECG by:

$$
\overrightarrow{V C G}(t)=\sum_{e l=1}^{9} R^{2} e c g_{e l}(t) \cdot|\vec{r}| \quad e q .1
$$

where $\vec{r}=\left|\overrightarrow{r_{e l}}-\overrightarrow{r_{r e f}}\right|$ is the normalized vector between a reference position and the electrode position on the thorax and $\mathrm{R}$ the length of $\vec{r}$. The reference position was set to coincide with the center of ventricular mass (CVM), close to left ventricular septum. The $e c g_{e l}(t)$ is the value at of the ECG at an electrode at sample t.

The next step is to compute the meanTSI from this VCG signal. Assuming the activation starts at are near the CVM the mean path of the activation can be obtained. The activation path direction is based on the VCG direction per time sample, using an propagation velocity of $0.7 \mathrm{~m} / \mathrm{s}$ through the myocardium [3].

This meanTSI is a representation of the mean position of the propagating 3D activation wave through the myocardium. When assuming a constant and homogeneous myocardial activation velocity the change in vector position should approximately move with this velocity in the direction indicated by the VCG signal. Since all patients were in sinus rhythm, the meanTSI starting location is represented by the CVM. ACM is characterized by activation delay, most frequently beyond the normal QRS duration. Therefore, the first $25 \mathrm{~ms}$ after the QRS ends might reveal a delayed activation direction visible in the meanTSI. Despite the limited amplitude of the ST segment, a direction can be obtained comparable with the initial vector directions of a PVC.

The final step is to directly relate and visualize the meanTSI movement into the 3D heart space. For this purpose the software automatically determines the LeftRight (LR) axis, the Base-Apex (BA) axis, and the Posterior-Anterior (PA) axis from the heart model. From these axes the clinical relevant planes: the 4 chamber view, the LAO and RAO planes can be defined, see Figure 1. For the current study a standard heart model was used for all patients. 


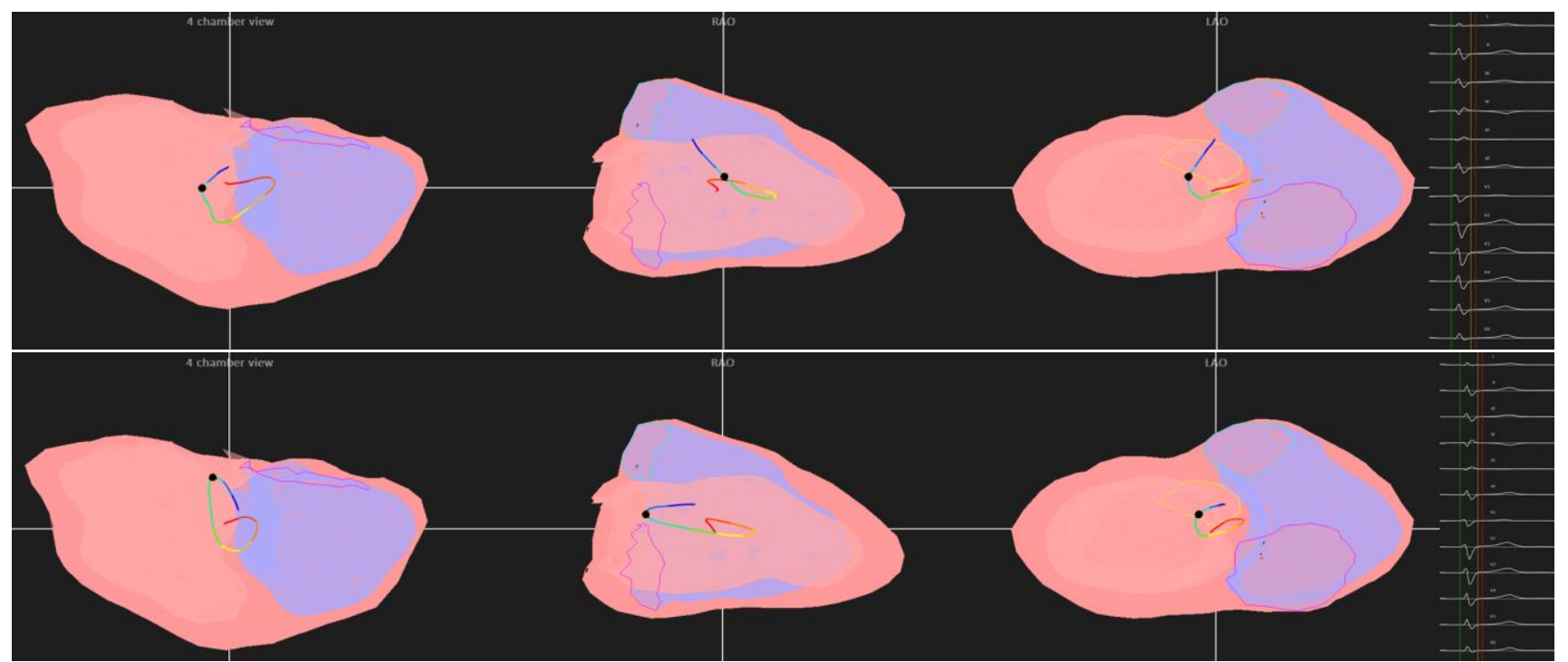

Figure 2: The mean TSI of a typical mutation carrier patient (top row) and a patient from the control group (bottom row). The Mean TSI starts in the center of mass of the heart (red line) and ends in dark blue, 25 ms after the end of the QRS. The end of the QRS is indicated by a black dot on the mean TSI trajectory. On the right the 12 lead ECG is shown. The carrier patient the mean TSI moves dominantly in the basal plane, whereas in the control patient the mean TSI moves towards the apex. Planes are described in fig. 1.

Table 2. MeanTSI movement per heart axis and plane.

\begin{tabular}{lccccc}
\hline $\begin{array}{l}\text { Para } \\
\text { meter }\end{array}$ & $\begin{array}{c}\text { Carrie } \\
\mathbf{r}(\mathbf{n}=6)\end{array}$ & $\begin{array}{c}\text { ACM } \\
\mathbf{Q R S}<\mathbf{1 2 0} \\
(\mathbf{n}=10)\end{array}$ & $\begin{array}{c}\text { ACM } \\
\mathbf{Q R S}>\mathbf{1 2 0} \\
(\mathbf{n}=\mathbf{6})\end{array}$ & $\begin{array}{c}\text { Cont } \\
\mathbf{r o l} \\
(\mathbf{n}=6)\end{array}$ & $\begin{array}{c}\mathbf{P} \\
\text { value }\end{array}$ \\
\hline LR & $0.64 \pm$ & $0.44 \pm$ & $0.40 \pm$ & $0.80 \pm$ & 0.494 \\
& 0.27 & 0.24 & 0.26 & 0.26 & \\
PA & $0.45 \pm$ & $0.49 \pm$ & $0.27 \pm$ & $0.22 \pm$ & 0.494 \\
& 0.28 & 0.21 & 0.21 & 0.14 & \\
BA & $0.49 \pm$ & $0.65 \pm$ & $0.78 \pm$ & $0.84 \pm$ & 0.094 \\
& 0.15 & 0.20 & 0.20 & 0.20 & \\
relBP & $0.69 \pm$ & $0.59 \pm$ & $0.45 \pm$ & $0.41 \pm$ & 0.013 \\
& $0.09^{*}$ & 0.14 & 0.18 & $0.17 *$ & \\
relBA & $0.31 \pm$ & $0.41 \pm$ & $0.55 \pm$ & $0.59 \pm$ & 0.013 \\
& $0.08^{*}$ & 0.14 & 0,18 & $0.16^{*}$ & \\
\hline
\end{tabular}

Carrier: asymptomatic mutation carrier, ACM: arrhythmogenic cardiomyopathy. LR: Left-Right axis, PA: Posterior-Anterior axis, BA: Basal-Apex axis, relBP: relative Basal plane movement, relBA: relative BasalApex plane movement. ${ }^{*}$ statistical significant.

In the early phase of the ACM the anticipated delayed activation of the right free wall is hardly visible in the ECG. The right ventricular base and free wall are represented at the end of the QRS complex. Therefore, the delayed activation should most likely occur after the end of the QRS. In this study the $25 \mathrm{~ms}$ after the end of the QRS was analyzed using the meanTSI.

For every patient the complete meanTSI was computed, but only the interval between the QRS end and QRS end $+25 \mathrm{~ms}$ was used to quantify the direction in the 3 specified clinical axes (Figure 1). To quantify the movement in the base plane, the relative movement is computed and compared to the relative movement in the long axis heart plane. The relative base plane movement is defined as the movement in either the LR axis and the PA axis divided by the sum of the movement in all 3 axis. The remainder is the movement along the $\mathrm{BA}$ axis.

\subsection{Statistics}

Data were presented as mean \pm standard deviation or median (25th-75th percentile) as appropriate. Normality of the data was assessed due to inspection of histograms and Q-Q plots. Normal distributed continuous data was analyzed using the One-Way ANOVA.. A 2-sided P value of $<0.05$ was considered significant. Statistical analysis was performed using SPPS (ver. 25).

\section{Results}

The principal direction of the meanTSI $25 \mathrm{~ms}$ after end QRS in ACM patients and the controls was found in the base apex axis. For the asymptomatic mutation carriers the principal movement of the meanTSI was found in the basal plane (relBA and relBP in table 2). ACM patients with normal QRS duration had more movement in the base plane compared to the ACM patients with prolonged QRS, which was comparable to the control group. This control group also had significantly more BA movement compared to mutation carriers $(\mathrm{p}=0.013)$ and moved less in the base plane $(\mathrm{p}=0.013)$.

Two representative examples of a control and mutation carrier patient are shown in figure 2 . 


\section{Discussion}

This feasibility study shows that the meanTSI, derived from the 12 lead ECG, might be used as a screening method to quantify the activation delay progression in patients with ACM and asymptomatic mutation carriers. The study included a clinical relevant cohort with subgroups of different stages of activation delay.

The main findings of the study were that asymptomatic mutation carriers showed significantly more meanTSI movement in the basal plane (relBP, table 2). The relative movement directions in ACM patients with prolonged QRS and controls were very similar. For controls, this is most probably representing solely the initial phase of the repolarization. In contrast, ACM patients with a prolonged QRS probably have severe activation delay and $\mathrm{RV}$ dilation. The importance of these meanTSI directions should be a subject for further studies. ACM patients with a normal QRS duration showed more basal plane movement than the later 2 groups, but less than the asymptomatic mutation carriers.

The difference in meanTSI movement between the mutation carriers and the identified ACM patients with normal QRS duration might be related to the difference in affected substrate. The patients already diagnosed with ACM have already significant electrical and structural changes, whereas the carrier patients might only have subtle activation delay due to fibrofatty replacement and desmosome dysfunction. Previous studies have shown that electrical changes are the early signs of ACM development. [5] Adding the meanTSI to the standard screening of asymptomatic mutation carriers with the ECG is feasible and could aid in the early detection of local activation delay.

Although the number of patients in this feasibility study is limited, the study indicates that some of the anticipated features are still detectable in the ECG. Additional studies with larger patient groups are required to validate the meanTSI characteristics in ACM. Especially the group of mutation carriers needs to be followed for a longer time period, so development and progression of the disease can be monitored and correlated with this novel marker. This might shed light on the structural differences between mutation carriers and ACM patients.

\subsection{Limitations}

The used planes to project the meanTSI are dependent on the patient specific orientation and position of the heart in the thorax. For this study, we used an standard heart and thorax model, which influences the results of this study. Moreover, in patients with advanced stages of ACM the right ventricle is enlarged and the myocardium is locally replaced with fibrofatty tissue. This problem could be solved by using patient specific heart models derived from MRI- or CT-scans.

Furthermore, we require more knowledge on the activation propagation in ACM patients. Because, the meanTSI assumes a constant myocardial activation velocity. Previously performed electro-anatomical mapping studies in patients with ACM have shown that there are large areas with local activation delay primarily on the epicardial side of the right ventricle and delay in endocardial to epicardial activation. [6]

\section{Conclusion}

The mean TSI based on the interval directly after the end of the QRS complex has potential to become a screening method to quantify the activation delay in patients with ACM and asymptomatic mutation carriers in a non-invasive manner. Controls differed from asymptomatic mutation carriers based on base to apex movement and relative basal movement.

\section{Acknowledgements}

This study was funded by the European Commission through the Eurostars program, grant number 9799 (ALVALE project: Anatomical Localization of the origin of Ventricular Arrhythmias from the 12-Lead ECG) and the Dutch Heart Foundation, grant number CVON201512 (eDETECT: Early detection of disease in cardiomyopathy mutation carriers). Peter van Dam is the owner of Peacs BV (the Netherlands).

\section{References}

[1] Corrado D, Basso C. Arrhythmogenic Cardiomyopathy. Circ Res. 2017;15:784-802.

[2] Bhonsale A, James CA. Risk stratification in arrhythmogenic right ventricular dysplasia/cardiomyopathy associated desmosomal mutation carriers. Circ Arrhythm Electrophysiol 2013;6:569-578.

[3] van Dam PM. A new anatomical view on the vector cardiogram: The mean temporal-spatial isochrones. J Electrocardiol. 2017;50:732-738.

[4] Marcus FI, McKenna WJ. Diagnosis of arrhythmogenic right ventricular cardiomyopathy/dysplasia: proposed modification of the Task Force Criteria. Eur Heart J. 2010

[5] Mast TP, Teske AJ.Prolonged Electromechanical Interval Unmasks Arrhythmogenic Right Ventricular Dysplasia/Cardiomyopathy in the Subclinical Stage. J Cardiovasc Electrophysiol. 2016;27:303-14..

[6] Haqqani HM, Tschabrunn CM. Layered activation of epicardial scar in arrhythmogenic right ventricular dysplasia: possible substrate for confined epicardial circuits. Circ Arrhythm Electrophysiol. 2012;5:796-803

Address for correspondence.

Rob Roudijk

Heidelberglaan 100, 3584 CX, Utrecht, The Netherlands

E-mail address: r.w.roudijk@umcutrecht.nl 